\title{
A Single Layer Dual-Polarization Printed Bow-Tie Slot Broadband Antenna with C-Band Stop
}

\author{
${ }^{1}$ CHANG-JU WU, ${ }^{2}$ I-FONG CHEN, ${ }^{2}$ CHIA-MEI PENG, ${ }^{1}$ JWO-SHIUN SUN \\ ${ }^{1}$ Department of Electronic Engineering National Taipei University of Technology 1, Sec. 3, \\ Zhongxiao E. Road, Taipei 10608 TAIWAN, R.O.C \\ ${ }^{2}$ Institute of Computer and Comm. Engineering Jinwen University of Science and Technology No. 99, \\ An-Chung Road, New Taipei City 231 TAIWAN, R.O.C.
}

\begin{abstract}
In this letter, a single layer dual-polarization printed bow-tie slot broadband antenna with C-band stop is presented. The proposed antenna consists of two pairs of mirror-symmetric and mutually perpendicular bowtie slots with two coplanar waveguide (CPW)-fed. The proposed antenna has a very simple structure and the measured results demonstrate the antenna performance can be achieved a wide impedance bandwidth $79.1 \%$ for $|\mathrm{S} 11|$ and $|\mathrm{S} 22| \leqq-10 \mathrm{~dB}$ from $1.3 \sim 3 \mathrm{GHz}$ and $62.3 \%$ from $5.25 \sim 10 \mathrm{GHz}$, which has been implemented the operating band for GPS $(1575 \mathrm{MHz})$, WLAN band $(2.4 \mathrm{GHz}$ and $5.8 \mathrm{GHz})$, and $6 \mathrm{GHz}$ unlicensed spectrum $(5.925$ $\sim 7.125 \mathrm{GHz}$ ), UWB band (3.1 10.6 GHz), LTE Upper band (LTE, $1710 \sim 2690 \mathrm{MHz}$ ) applications. The measured in-band isolation performance between the two ports $|\mathrm{S} 21| \leqq-15.5 \mathrm{~dB}$ from $1.4 \sim 3 \mathrm{GHz}$, and $|\mathrm{S} 21|$ $\leqq-21 \mathrm{~dB}$ from $5 \sim 10 \mathrm{GHz}$ is significantly achieved and the radiation pattern, peak gain, and efficiency of the proposed antenna are measured as well. In the end, the simulated and measured radiation patterns are compared.
\end{abstract}

Key-Words: - Broadband, Dual Polarization, Isolation, Bow-Tie Slot, Band Stop.

Received: March 5, 2021. Revised: April 20, 2021. Accepted: April 28, 2021. Published: May 5, 2021.

\section{Introduction}

With rapid progress in wireless communication systems, the demand to enhance the information accessibility and wideband utility has become indispensable option in wireless technology. An efficient way to increase the capability is the employment of polarization diversity, and thus the dual polarized antennas have gained more and more popularities. In order to fulfill the demand, wideband and multiband antennas are widely utilized in wireless communication systems to cover multiple communication bands, such as Mobile Wireless Communication system $(2 \mathrm{G} / 3 \mathrm{G} / 4 \mathrm{G})$ bands operate at 698-960 MHz/1710-2690MHz, wireless local area network (WLAN) bands operate at 2.4-2.484 $\mathrm{GHz} / 5.15-5.825 \mathrm{GHz}$ and $6 \mathrm{GHz}$ unlicensed spectrum for the European markets is $5.925 \mathrm{GHz}$ to $6.425 \mathrm{GHz}$, and for the US markets between 5.925 $\mathrm{GHz}$ to $7.125 \mathrm{GHz}$, even the Ultra-wideband band (UWB) from $3.1 \sim 10.6 \mathrm{GHz}$. To be efficiently increased our research, the antenna design consideration will be simple structure, wideband and dual polarization. A few of papers [1-2] have been published for getting better isolation in some of dual polarization antenna applications, but the two proposed antennas of the impedance bandwidth are still not wide enough to cover modern mobile and wireless communication systems. For the purpose of a wider operating bandwidth and simple planar antenna configuration, bow-tie dipole and slot antenna structure are good candidates in reference research [3-5]. In the paper [6-7], a dual-polarization CPW-fed bow-tie slot antenna has been demonstrated, the antenna structure is simplicity and the specifications of wide bandwidth, high isolation that can be accomplished easily. In the current wireless application scenarios, broadband antenna with band-stop design is the mainstream, which will be more feasible for modern wireless applications [89]. UWB-MIMO antenna with multiple bandnotched design has attracted extensive attentions and researches in recent years [10-12], but its band coverage needs to consider lower band for fulfilling modern wireless applications.

In this communication, a single layer dualpolarization printed bow-tie slot broadband antenna with C-band stop is demonstrated. The proposed antenna can be achieved a wide impedance 
bandwidth $\mid$ S11 $\mid$ and $\mid$ S22 $\mid \leqq-10 d B$ for lower band $(1.3 \sim 3 \mathrm{GHz})$ and upper band $(5.25 \sim 10 \mathrm{GHz})$. The in-band isolation between the two input ports can be achieved $|\mathrm{S} 21| \leqq-15.5 \mathrm{~dB}$ for lower band, and $\mathrm{S} 21 \mid \leqq-21 \mathrm{~dB}$ for upper band. Details of the proposed antenna of the simulation and experimental results are analyzed and its performances are given in the next two sections.

\section{Antenna Structure and Design}

\subsection{Antenna Configuration}

Fig. 1 shows the top view of the configuration of the proposed a single layer dual-polarization printed bow-tie slot broadband antenna with C-band stop. The antenna structure is composed of two pairs of mirror-symmetric and mutually perpendicular bowtie slots with two coplanar waveguide (CPW)-fed. The proposed antenna overall dimension is $150 \times 150$ $\mathrm{mm}^{2}$ and the antenna pattern is printed on a thickness of $1.6 \mathrm{~mm}$ FR4 glass epoxy substrate (the relative permittivity is 4.4 and Loss tangent is 0.02 ). There are two $45 \times 44 \mathrm{~mm}^{2}$ equilateral right triangle slots on the lower left and upper right of the antenna, which are part of the antenna radiator. The middle equilateral right triangle slot of the antenna radiator is $63.8 \times 63.8 \mathrm{~mm}^{2}$ and a short-circuit microstrip (d1 $=12 \mathrm{~mm}$ ) is used in the middle of the slot to construct a pair of mirror-symmetric equilateral triangle slots for better isolation between the two antennas. Two pairs of symmetrical stub slots $(\mathrm{d} 2=11 \mathrm{~mm}, \mathrm{~L} 3=$ $13 \mathrm{~mm}, \mathrm{~S} 1=2 \mathrm{~mm}$ ) are cut out over the antenna body. The structure can be considered as a $\pi$-type filter circuit model, which is perpendicular to each other for tuning the C-band stop with dual-polarization. The CPW-fed line is designed to be $50 \Omega$ (L3 = $3.2 \mathrm{~mm})$, gap spacing $(\mathrm{g}=0.2 \mathrm{~mm})$, and the tapered signal microstrip length of the CPW structure is recommended $0.175 \lambda(\mathrm{W} 2=35 \mathrm{~mm})$ for broadband impedance matching. The CPW-fed in port 1 is located on the lower side of the antenna radiator, it serves as the vertical polarization radiation. In the upper right side of the antenna radiator, the antenna is rotated $90^{\circ}$ with the $\mathrm{CPW}$-fed in port 2 to cover the horizontal polarization radiation. All the parameters can be determined by the desired band of the lowest frequency and the final optimized geometric parameters of the antenna are listed in Table I.

Table I. Dimensions of the proposed antenna.

\begin{tabular}{|l|l|l|l|l|l|l|}
\hline Parameter & L & L1 & L2 & L3 & Ls & S1 \\
\hline
\end{tabular}

\begin{tabular}{|l|c|c|c|c|c|c|}
\hline Value(mm) & 150 & 35 & 3.2 & 13 & 43.8 & 2 \\
\hline Parameter & S2 & S3/S4 & h & W & W1 & W2 \\
\hline Value(mm) & 1.2 & 0.5 & 1.6 & 150 & 85 & 63.8 \\
\hline Parameter & W3 & Ws & d1 & d2 & g & $\theta$ \\
\hline Value $(\mathrm{mm})$ & 65.6 & 43.8 & 12 & 11 & 0.2 & $45^{\circ}$ \\
\hline
\end{tabular}

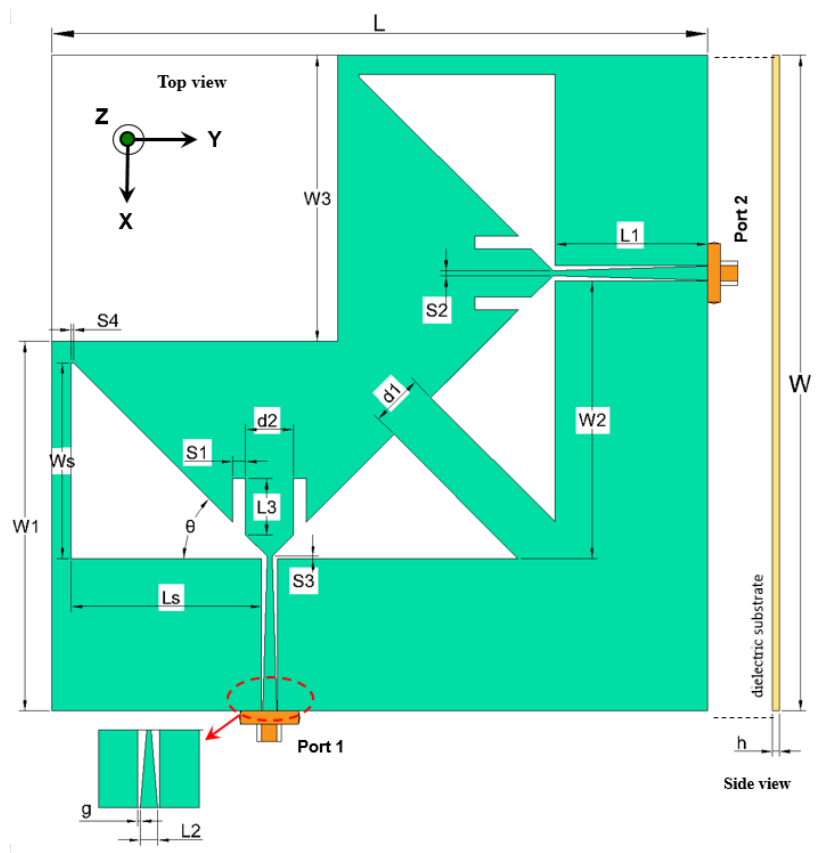

Fig. 1 Geometry of the dual-polarization bow-tie slot broadband antenna with C-band stop.

\subsection{Antenna Design Guideline}

In the beginning, we studied the antenna structure indepth in the reference [3][6-7] and simulated using Ansys High Frequency Structure Simulator(HFSS) to verify the wideband performance of these antennas. It's observed the lowest operating frequency can be estimated by the following equation:

$$
f_{L}=\frac{c}{\lambda_{L}} \cong \frac{C}{\text { Slot length }} \times 0.75 \quad(G H z)
$$

where $\mathrm{C}$ is the speed of light and Slot length represent the total length of a right triangle slot edge. According to the formulation, the dual-polarization printed bow-tie slot broadband antenna of a right triangle slot edge total length can be approximately calculated. Take frequency $1.5 \mathrm{GHz}$ as an example, when $f_{L}=1.5 \mathrm{GHz}$, the calculated total length of a right triangle slot is $150 \mathrm{~mm}$. The calculated result is roughly consistent with the paper [6][7], the total length of the right triangle slot is $151 \mathrm{~mm}$ and $140.8 \mathrm{~mm}$, respectively. 
Fig. 2 shows the simulated reflection coefficient and isolation performance of the two references. As a result, the two antennas structure can clearly meet the lowest and highest frequency requirements of the desired broadband. In order to effectively increase the dual-polarization broadband antenna practicality in MIMO wireless applications. The proposed antenna is based on the reference [6] as the prototype, and two pairs of symmetrical stub slots are cut out on the antenna body to implement the feature of the Cband stop with dual-polarization. The parametric studies and results will be demonstrated in the next section.

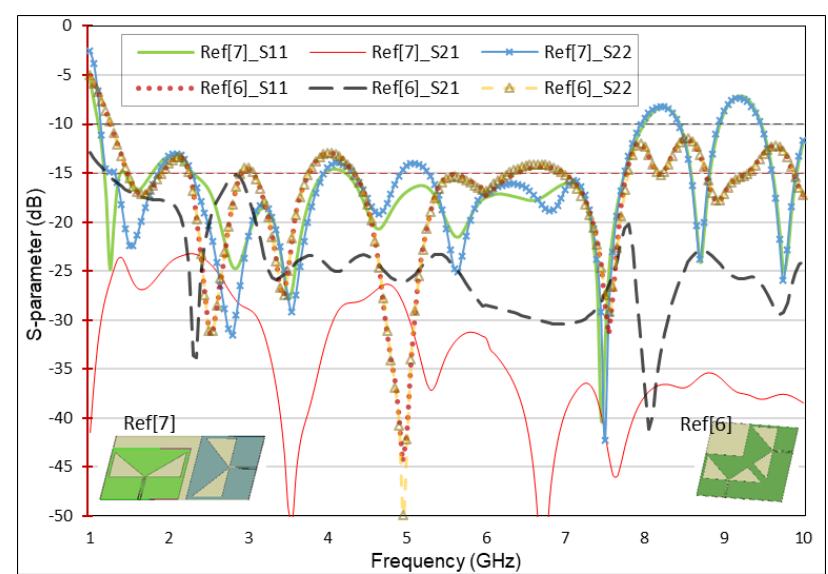

Fig. 2 The simulated reflection coefficient and isolation performance of the two references.

\subsection{Parametric Studies}

The key parameters L3, d2, and S1 will dominate the main frequency band stop and bandwidth performance.

Fig. 3 shows the relationship between the parameter L3. It can be seen when L3 $=13.5 \mathrm{~mm}$, the reflection coefficient has the highest impedance mismatch at $3.6 \mathrm{GHz}$, which is matched our original research purpose to perform C-band stop among the two input ports. The parameter L3 becomes shorter or longer, the highest impedance mismatch will move to higher or lower frequencies and compared with the $f_{L}$, the $\lambda_{L}$ can be approximated to be $0.065 \lambda$.

Fig. 4 shows the relationship between the parameter $\mathrm{d} 2$. It is observed when $\mathrm{d} 2$ becomes shorter ( $\leqq 9 \mathrm{~mm}$ ), the starting frequency of impedance mismatch will move to a lower frequency and the bandwidth will become worse. When $\mathrm{d} 2$ is equal or bigger than $11 \mathrm{~mm}$, the starting frequency of impedance mismatch will move to a higher frequency, but the bandwidth is almost the same.

Fig. 5 shows the relationship between the parameter S1. As a result, it is well known the two pairs of symmetrical stub slots can be equivalent to a $\pi$-type filter circuit model, when S1 becomes wider (C value is larger), the starting frequency of impedance mismatch is almost kept, but the stopping frequency will move to a higher frequency.

A Summary of the simulation results of the proposed antenna, the parameters $\mathrm{L} 3=13 \mathrm{~mm}, \mathrm{~d} 2=$ $11 \mathrm{~mm}, \mathrm{~S} 1=2 \mathrm{~mm}$ can completely achieve the $\mathrm{C}$-band stop feature with better performance.

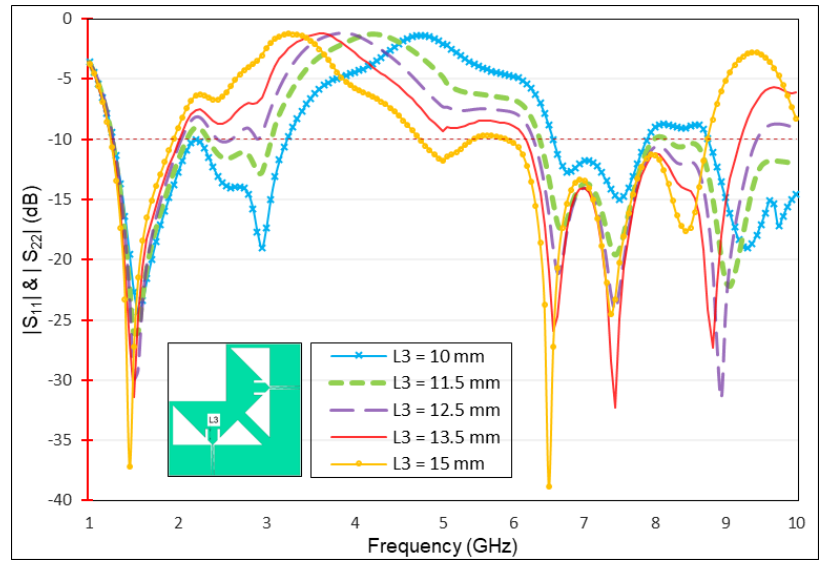

Fig. 3 Simulated for the parameter L3 reflection coefficient versus frequency.

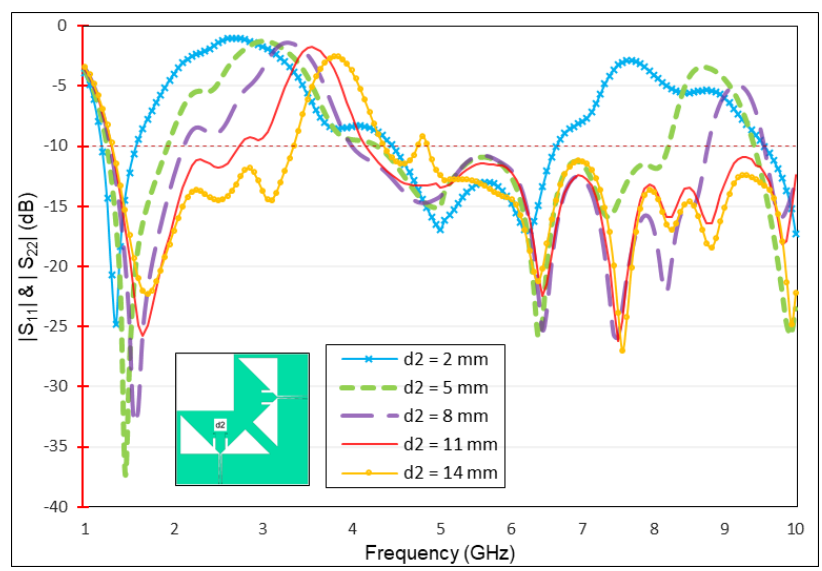

Fig. 4 Simulated for the parameter $\mathrm{d} 2$ reflection coefficient versus frequency. 


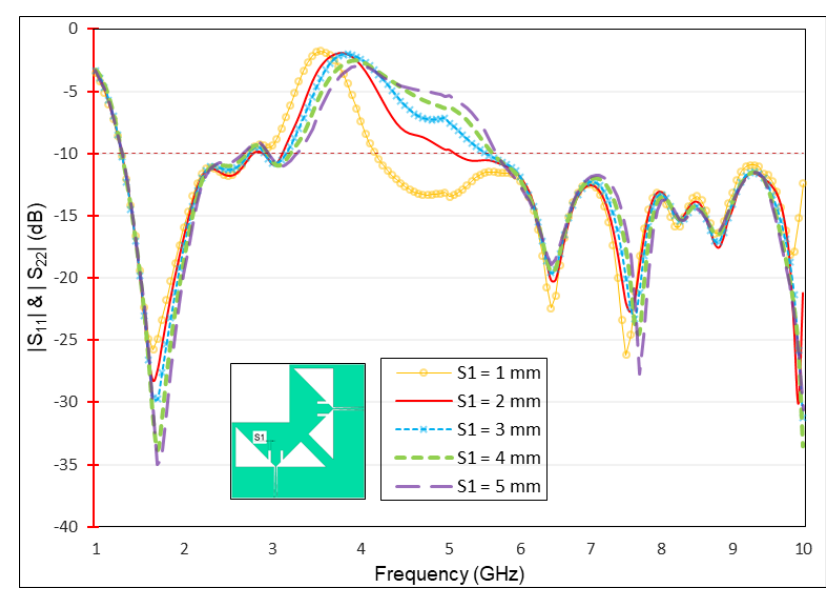

Fig. 5 Simulated for the parameter S1 reflection coefficient versus frequency.

\section{Simulated and Measured Results}

\subsection{S-parameters with Prototype}

The proposed antenna prototype has been fabricated with an eagle view as shown in Fig. 6. Meanwhile, the simulation and measurements were carried out to demonstrate the performances.

Fig. 7 demonstrates the simulated and measured impedance bandwidth results for $|\mathrm{S} 11|$ and $|\mathrm{S} 22| \leq$ $-10 \mathrm{~dB}$. For lower band, the simulated results on two ports are almost the same $83 \%$ from $1.26 \sim 3.06 \mathrm{GHz}$, and the measured results are $86.8 \%$ for port- 1 and $84.9 \%$ for port- 2 from $1.2 \sim 3.04 \mathrm{GHz}$. For the upper band of $5 \sim 10 \mathrm{GHz}$, the simulated results on two ports are $67.2 \%$ and $66.7 \%$, respectively. The measured results are pretty much the same, being $62.3 \%$ on port- 1 and port-2. The lowest notched frequency is $3.75 \mathrm{GHz}$, where the return loss is around $-1.8 \mathrm{~dB}$. We observed the prototype measured result has a slight frequency deviation compared with the simulated result at $5 \mathrm{GHz}$ that is because of the PCB board making error.

Fig. 8 demonstrates the simulated and measured in-band isolation between the two input ports $\mid$ S21 $\leqq-15.5 \mathrm{~dB}$ from $1.27 \sim 3.15 \mathrm{GHz}$, and $|\mathrm{S} 21| \leqq$ $-21 \mathrm{~dB}$ from $3.16 \sim 10 \mathrm{GHz}$ are obtained.

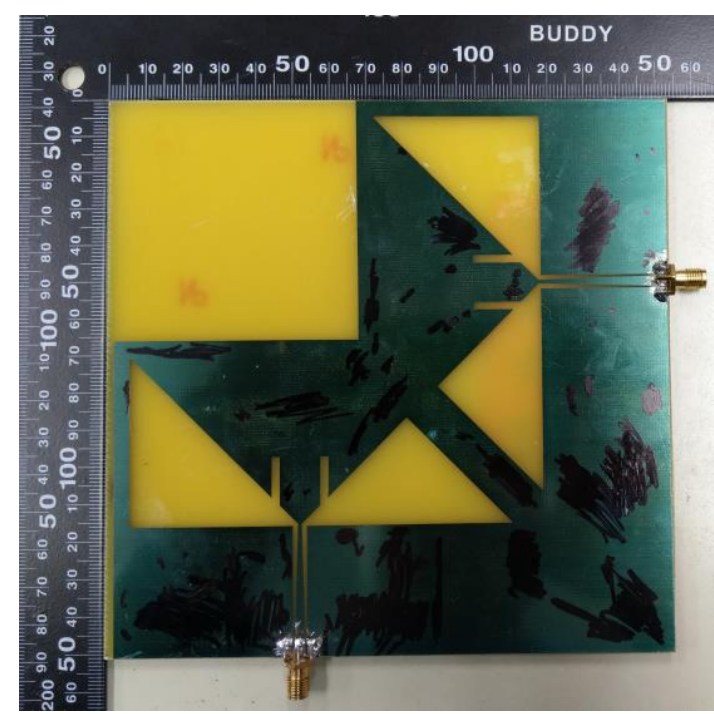

Fig. 6 Implemented a prototype of the proposed antenna.

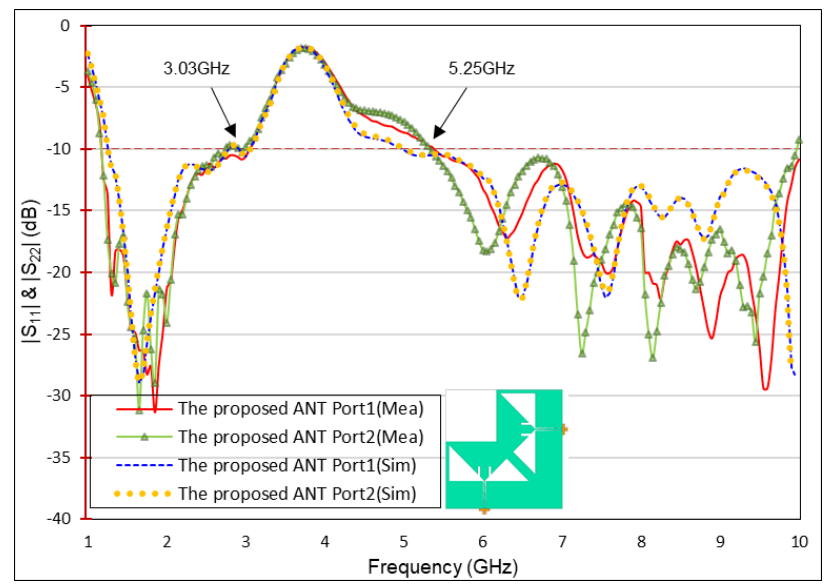

Fig. 7 Measured and simulated reflection coefficient versus frequency of the proposed antenna.

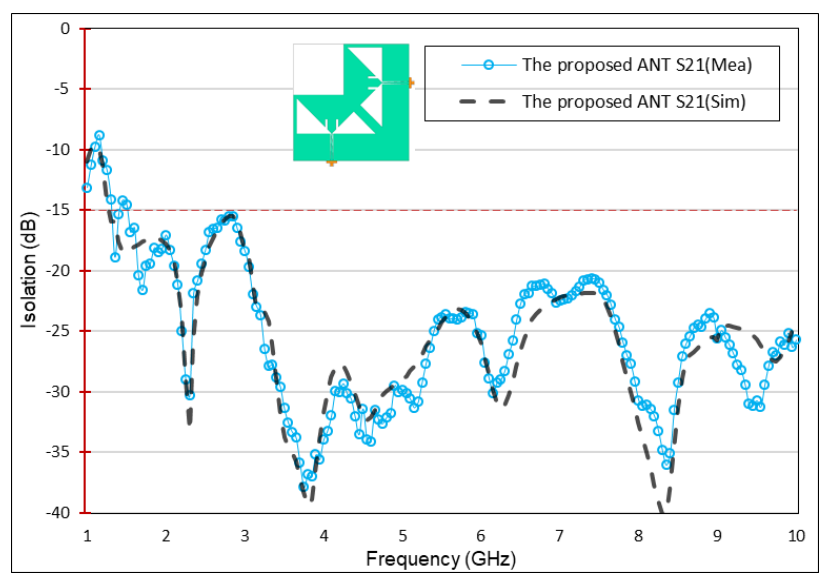

Fig. 8 Measured and simulated isolation versus frequency of the proposed antenna. 


\subsection{Radiation Patterns, Peak Gain and Efficiency}

The proposed antenna supports dual-polarization is the main purpose, so the $2 \mathrm{D}$ radiation pattern of the xz-plane at port-1 and yz-plane at port-2 should be similar to what is expected. When the port- 1 and port2 is excited, the radiation patterns for free space of the proposed antenna are shown in Fig. $9 \sim 12$. For port-1, the vertical polarization is the dominant polarization. The half-power(3-dB) beamwidths are $90^{\circ}$ and $50^{\circ}$ in E1-plane (xz-plane) and E2-plane (yzplane), the maximum radiation power(MRP) is concentrated at about 0 degree on the plus $\mathrm{Z}$-axis at $1.575 \mathrm{GHz}$. For port-2, the horizontal polarization is the dominant polarization. The $3-\mathrm{dB}$ beamwidths are $40^{\circ}$ and $50^{\circ}$ in xz-plane and yz-plane, the MRP is concentrated on the same axis as port- 1 at $1575 \mathrm{MHz}$. At the frequency of $2.4 \mathrm{GHz}$, the $3-\mathrm{dB}$ beamwidths are $40^{\circ}$ and $60^{\circ}$ in $\mathrm{xz}$ - and yz- plane in port-1, and $50^{\circ}$ in xz- and yz- plane in port-2, the MRP of the two ports are the same as they are concentrated at about 0 degree on the plus $\mathrm{Z}$-axis. At $5.8 \mathrm{GHz}$ of port-1, the 3-dB beamwidths are $30^{\circ}$ in xz-plane and yz-plane, the MRP concentration between the plus X- and Zaxis is deviated at about 45 degrees. For port-2, the $3-\mathrm{dB}$ beamwidths are $40^{\circ}$ in xz-plane and $30^{\circ}$ in yzplane, the MRP concentration is on the same axis as port-1. From the perspective of $5 \mathrm{GHz}$ and higher frequency radiation patterns, when the operating frequency is higher than $5 \mathrm{GHz}$, the $3-\mathrm{dB}$ beamwidths are almost limited around 30 degrees or even smaller in $\mathrm{xz}$ - and yz- plane, and the MRP is scattering more seriously that is because the wavelength of $5 \mathrm{GHz}$ and higher frequency has tripled relative to the lowest frequency of the desired band.

Summarize the measured results of all radiation patterns in xz-plane and yz-plane, it is clearly observed that the proposed antenna radiation patterns are similar when feeding on port- 1 and port- 2 . The geometry of the proposed antenna will make the radiation patterns have obvious polarization diversity, and analyzed in E-plane results that can be proved the proposed antenna obtained both polarization diversity and pattern diversity characteristics with good isolation.

The 3D pattern efficiency and peak gain of the proposed antenna are also measured. The measurement result was done by using pattern integration employing the ETS-Lindgren anechoic chamber and it's shown in Fig. 15. In the GPS band of $1.575 \mathrm{GHz}$, the efficiency and peak gain are $81.6 \%$ and $4.4 \mathrm{dBi}$ for port- 1 and $77.8 \%$ and $4.8 \mathrm{dBi}$ for port2. In the WLAN band of $2.4 \mathrm{GHz}, 5.8 \mathrm{GHz}$ and $7.125 \mathrm{GHz}$, the efficiency are $62.6 \%, 87.8 \%$ and
$72.5 \%$ for port- 1 and $69.3 \%, 85.9 \%$ and $66.7 \%$ for port-2. The peak gain are 4.2, 4.3 and $5.5 \mathrm{dBi}$ for port-1 and 6.3, 3.9 and $3.9 \mathrm{dBi}$ for port-2. In the Cband of the center frequency $3.7 \mathrm{GHz}$, the efficiency and peak gain is $15.4 \%$ and $-2 \mathrm{dBi}$ for port- $1,18.7 \%$ and $-1.8 \mathrm{dBi}$ for port-2. The results demonstrate the C-band stop feature of the proposed antenna is perfectly achieved with better performance.

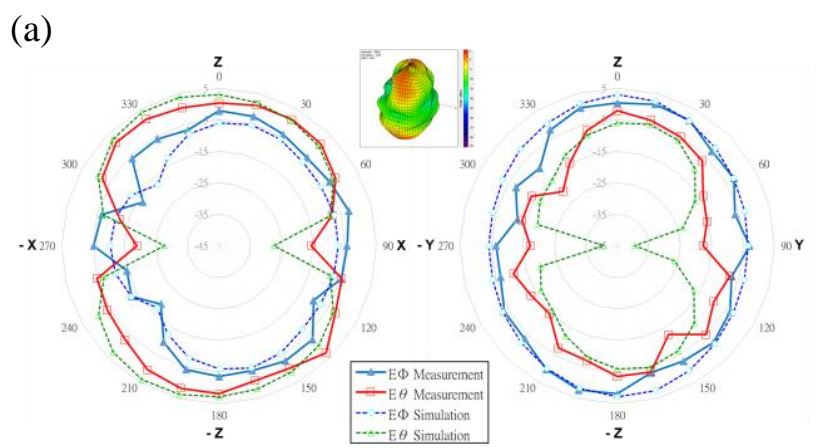

(b)

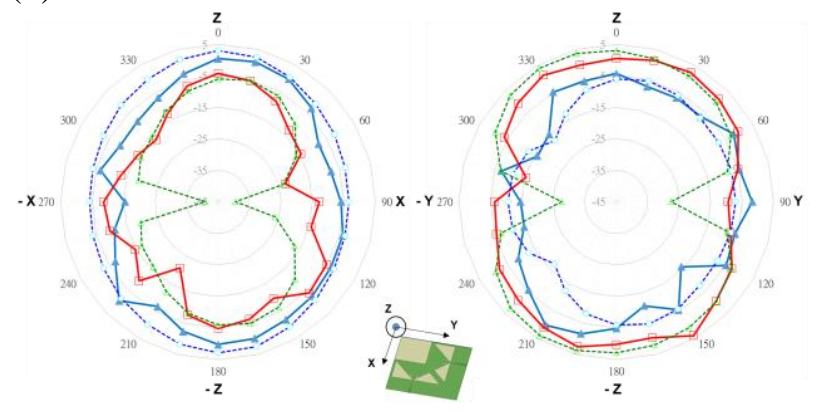

Fig. 9 Simulated and measured radiation patterns when feeding on xz-plane and yz-plane at $1.575 \mathrm{GHz}$ : (a) Port-1 (b) Port-2

(c)

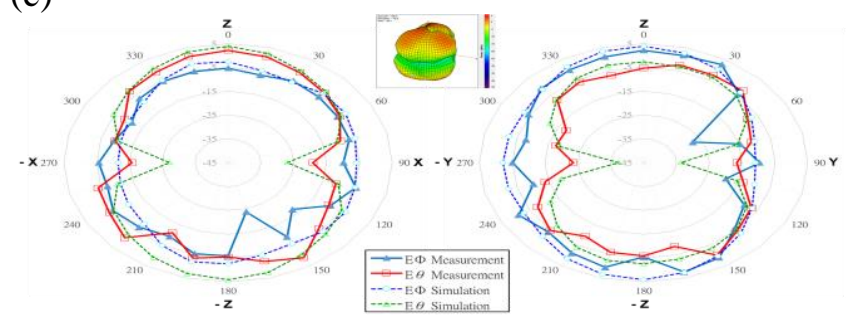


(d)
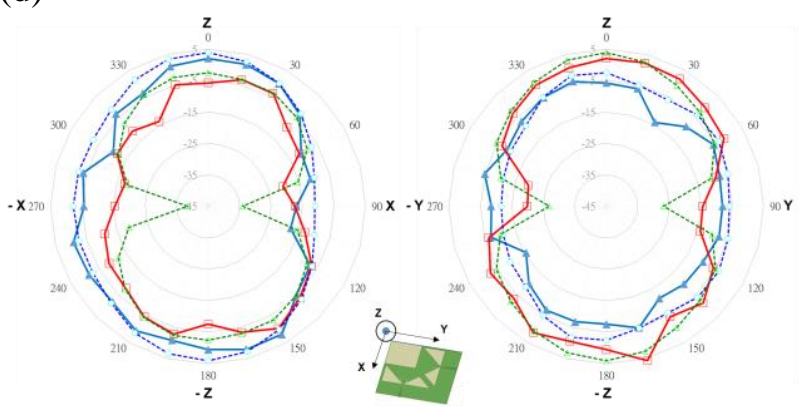

Fig. 10 Simulated and measured radiation patterns when feeding on xz-plane and yz-plane at $2.4 \mathrm{GHz}$ : (c) Port-1 (d) Port-2

(e)

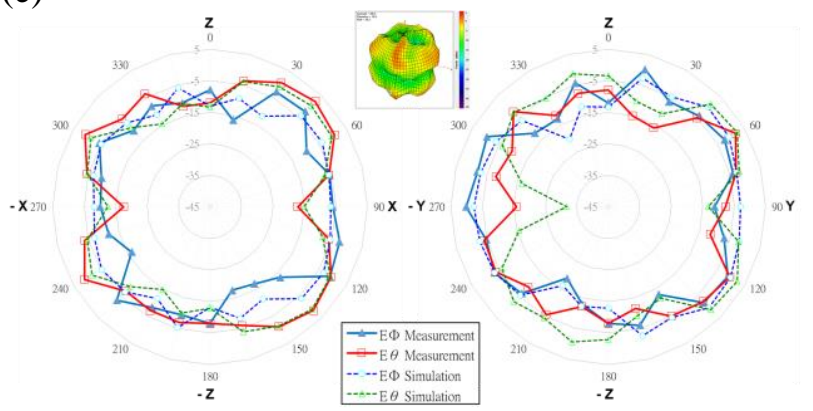

(f)

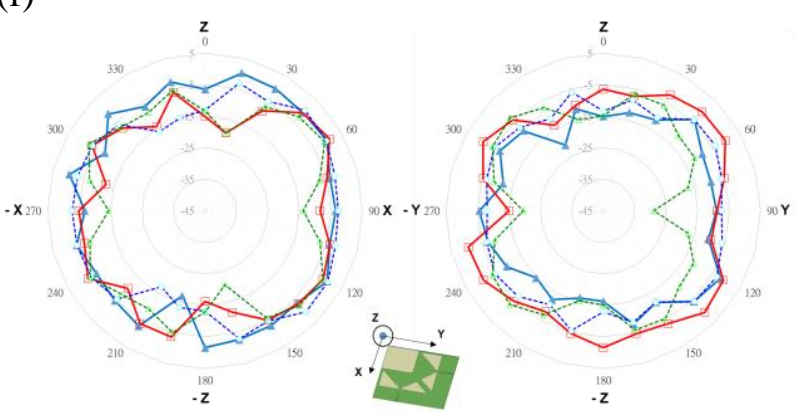

Fig. 11 Simulated and measured radiation patterns when feeding on xz-plane and yz-plane at $5.8 \mathrm{GHz}$ : (e) Port-1 (f) Port-2

(g)

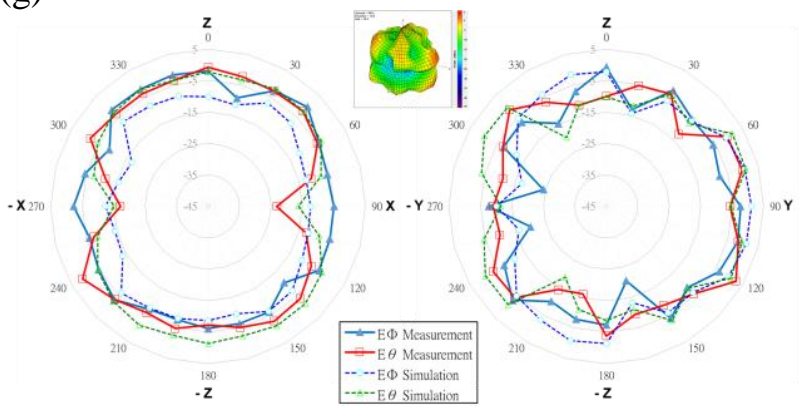

(h)

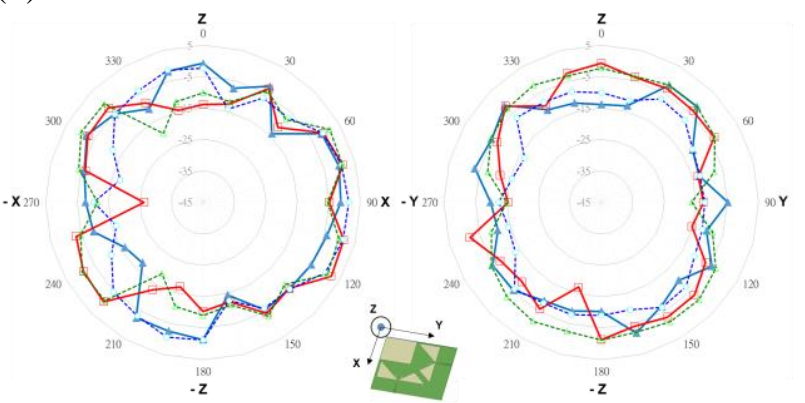

Fig. 12 Simulated and measured radiation patterns when feeding on xz-plane and yz-plane at $7.125 \mathrm{GHz}$ : (g) Port-1 (h) Port-2

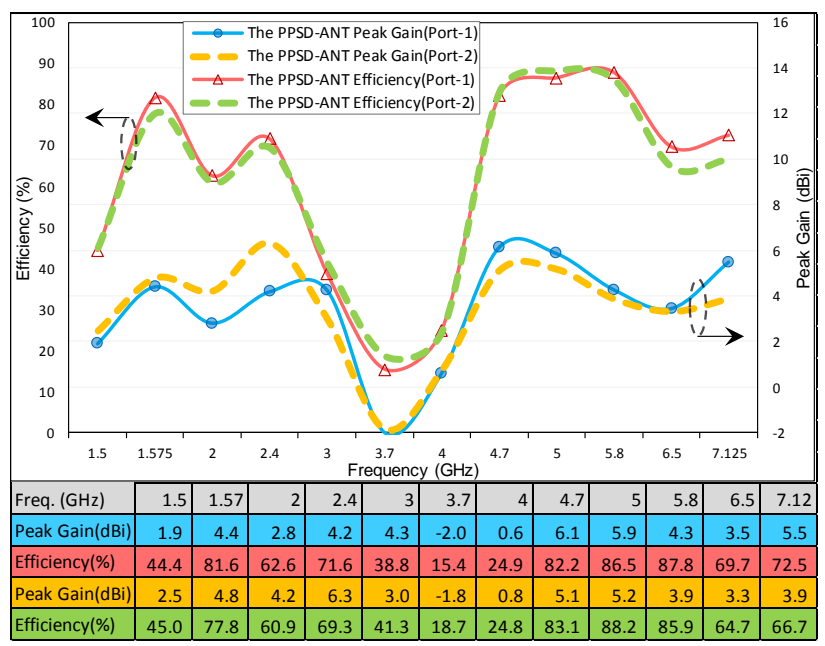

Fig. 13 Measured antenna peak gain \& efficiency versus frequency of the proposed antenna.

\subsection{Diversity Analysis}

To validate the MIMO capability and performance of the proposed antenna, the MIMO behavior is evaluated for the envelope correlation coefficient (ECC), diversity gain (DG), and total active reflection coefficient (TARC). The ECC can be evaluated using $\mathrm{S}$-parameters by the following relation [11-12].

$$
E C C=\frac{\left|S_{11}^{*} S_{12}+S_{21}^{*} S_{22}\right|^{2}}{\left(1-\left|S_{11}\right|^{2}-\left|S_{21}\right|^{2}\right)\left(1-\left|S_{22}\right|^{2}-\left|S_{12}\right|^{2}\right)}
$$

ECC should have ideally zero value but practical limit for an uncorrelated MIMO antenna is ECC < 0.5 . Fig. 14 shows the ECC of the proposed antenna has ECC less than 0.02 for the entire of the desired band. Another important parameter for evaluating MIMO antenna performance is diversity gain (DG). The DG of the MIMO antenna can be calculated by the following relation [12]. 


$$
D G=10 \sqrt{1-E C C^{2}}
$$

It is pointed out by Fig. 14, the proposed antenna has diversity gain greater than $9.97 \mathrm{~dB}$ with varying frequencies for the entire of the desired band.

The TARC is defined as the square root of the ratio of total reflected power to the total incident power and apparent return loss of the overall MIMO antenna system [11]. For dual-port MIMO system, it can be calculated as the following equation

$$
T A R C=\sqrt{\frac{\left(S_{11}+S_{12}\right)^{2}+\left(S_{21}+S_{22}\right)^{2}}{2}}
$$

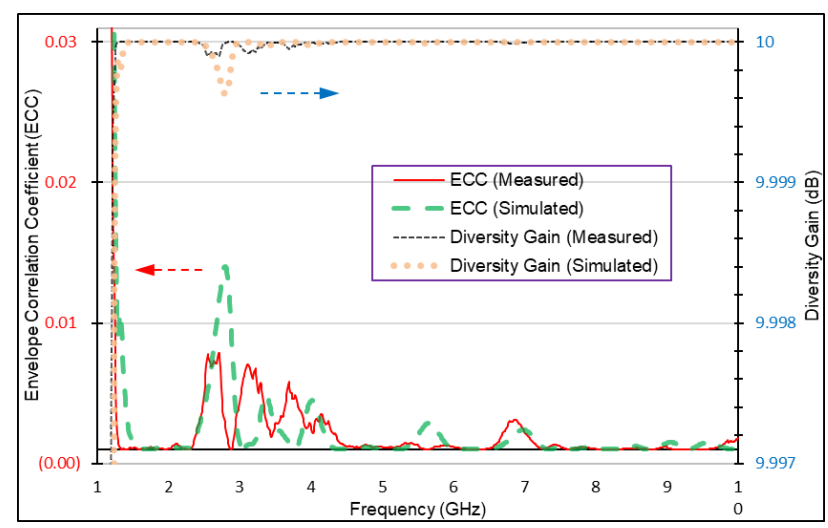

Fig. 14 Measured and simulated ECC and diversity gain.

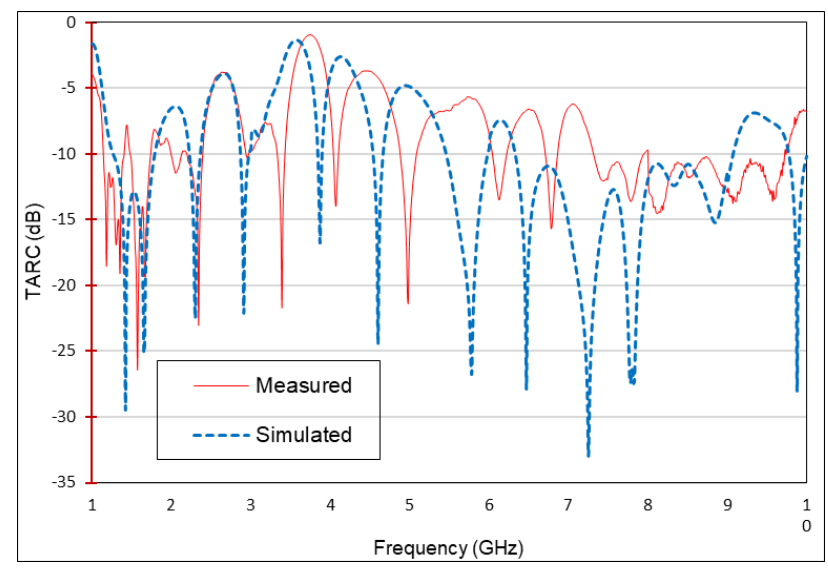

Fig. 15 TARC verses frequency for the proposed MIMO antenna.

It is shown in Fig. 15 that the TARC is less than $-4 d B$ for the desired band except for the C-band stop band.

\section{Conclusion}

A single layer dual-polarization printed bow-tie slot broadband antenna with C-band stop is proposed in this study. The antenna has a very simple structure and the measured results demonstrate the proposed configuration can be achieved a wide impedance bandwidth $|\mathrm{S} 11|$ and $|\mathrm{S} 22| \leqq-10 \mathrm{~dB}$ for lower band is $79.1 \%$ from $1.3 \sim 3 \mathrm{GHz}$, and for upper band is $62.3 \%$ from $5.25 \sim 10 \mathrm{GHz}$. The measured in-band isolation between the two input ports $|\mathrm{S} 21| \leqq-15 \mathrm{~dB}$ for lower band, and $|\mathrm{S} 21| \leqq-21 \mathrm{~dB}$ for upper band is significantly achieved. Alongside ECC $<0.02$, DG $>9.97 \mathrm{~dB}$, and TARC $<-4 \mathrm{~dB}$ are demonstrated that the proposed antenna has good performance over the proposed band.

\section{References:}

[1] C.-H. Lee, S.-Y. Chen, and P. Hsu, "Isosceles triangular slot antenna for broadband dual polarization applications," IEEE Trans. Antennas Propag., vol. 57, no. 10, pp. 3347-3351, Oct. 2009.

[2] Y. Li, Z. Zhang, W. Chen, Z. Feng, M.F. Iskander, "A dual-polarization slot antenna using a compact CPW feeding structure," IEEE Antennas Wireless Propag. Lett., vol. 9, pp. 191-194, 2010.

[3] Huang, C.Y., and Lin, D.Y.: "CPW-fed bow-tie slot antenna for ultrawideband communications," Electron. Lett., Vol. 42, No. 19, pp. 1073-1074, 2006.

[4] I.-F. Chen, C.-M. Peng, C.-C. Hung, and H.-C. Chen, "Printed modified bow-tie dipole antenna for multiband applications," IEICE Transactions on Communications, vol. E92.B, no. 4, pp. 1404-1405, 2009.

[5] Ming-Tien Wu, Ming-Lin Chuang, "Multibroadband Slotted Bow-Tie Monopole Antenna," IEEE Antennas and Wireless Propag. Lett., vol. 14, pp. 887-890, 2015.

[6] Chia-Mei Peng, I-Fong Chen, Chang-Ju Wu, "A single layer dual-polarization printed bow-tie broadband antenna," Proceedings of the 2012 IEEE International Symposium on Antennas and Propagation, 8-14 July 2012.

[7] Chang-Ju Wu, I-Fong Chen, Chia-Mei Peng, JwoShiun Sun, "A Dual Polarization Bow-Tie Slot Antenna For Broadband Communications," The International Journal of Engineering and Science (IJES), Volume 7, Issue 6 Ver. I, Pages 49-56, 201806.

[8] C.Y. Huang, C.C. Lin and W.F. Chen, "Multiple band-stop bow-tie slot antennas for multiband wireless systems", IET Microwaves Antennas and Propa., vol. 2, no. 6, pp. 588-593, 2008.

[9] Ching-Chih Hung, Chia-Mei Peng, and I-Fong Chen, "Printed Modified Bow-Tie Dipole Antenna for DVB/WLAN Applications," Hindawi International 
Journal of Antennas and Propagation, Volume 2013, Article ID 149296, 6 pages.

[10] Gao, P., S. He, Z. Xu, and Y. Zheng, "Compact printed UWB diversity slot antenna with $5.5-\mathrm{GHz}$ band-notched characteristics," IEEE Antenna and Wireless Propagation Letters, Vol. 13, 376-379, 2014.

[11] S. I. Jafri, R. Saleem, M. F. Shafique, and A. K. Brown, "Compact reconfigurable multiple-inputmultiple-output Antenna for ultra wideband applications," IET Microw., Antennas Propa., vol. 10, no. 4, pp. 413-419, Mar. 2016.

[12] Amjad Iqbal, Omar A. Saraereh, Arbab Waheed Ahmad, Shahid Bashir, "Mutual Coupling Reduction Using F-Shaped Stubs in UWB-MIMO Antenna," IEEE Access, vol. 6, pp. 2755 - 2759, Dec. 2017.

\section{Creative Commons Attribution License 4.0 (Attribution 4.0 International, CC BY 4.0)}

This article is published under the terms of the Creative Commons Attribution License 4.0

https://creativecommons.org/licenses/by/4.0/deed.en_US 